\title{
Mitochondria, oxidative stress and cell death
}

\author{
Martin Ott • Vladimir Gogvadze • Sten Orrenius • \\ Boris Zhivotovsky
}

Published online: 9 February 2007

(C) Springer Science + Business Media, LLC 2007

\begin{abstract}
In addition to the well-established role of the mitochondria in energy metabolism, regulation of cell death has recently emerged as a second major function of these organelles. This, in turn, seems to be intimately linked to their role as the major intracellular source of reactive oxygen species (ROS), which are mainly generated at Complex I and III of the respiratory chain. Excessive ROS production can lead to oxidation of macromolecules and has been implicated in mtDNA mutations, ageing, and cell death. Mitochondriagenerated ROS play an important role in the release of cytochrome $c$ and other pro-apoptotic proteins, which can trigger caspase activation and apoptosis. Cytochrome $c$ release occurs by a two-step process that is initiated by the dissociation of the hemoprotein from its binding to cardiolipin, which anchors it to the inner mitochondrial membrane. Oxidation of cardiolipin reduces cytochrome $c$ binding and results in an increased level of "free" cytochrome $c$ in the intermembrane space. Conversely, mitochondrial antioxidant enzymes protect from apoptosis. Hence, there is accumulating evidence supporting a direct link between mitochondria, oxidative stress and cell death.
\end{abstract}

Keywords Apoptosis - Cardiolipin · Cell death . Mitochondria $\cdot$ Oxidative stress

\section{Introduction}

Early in evolution, oxygen and its reactive metabolites were a major threat to the primitive eukaryotic cell. The reactive

M. Ott · V. Gogvadze · S. Orrenius $(\triangle) \cdot$ B. Zhivotovsky Institute of Environmental Medicine, Karolinska Institutet, Box 210, S-171 77 Stockholm, Sweden

e-mail: Sten.Orrenius@ki.se nature of the oxygen species made it necessary to develop defense mechanisms to protect cellular macromolecules from damage. With the dangerous side of oxygen controlled, molecular oxygen could be used to drive various essential biochemical reactions, among them the process of oxidative phosphorylation. However, under conditions when the formation of reactive oxygen species (ROS) is enhanced, they can still impair mitochondrial function and influence the viability of cells. Dependent on the impact, the cells can either repair the damage or activate pathways leading to cellular suicide. In fact, cells as different as neurons and yeast cells can die by a similar mode of cell death, when exposed to severe oxidative stress. The existence of an apoptosis-like cell death phenotype in yeast suggests that ROS are highly conserved signals to trigger cell death [1].

In humans, oxidative stress has been implicated in a wide variety of pathologies, including cancer, type II diabetes, arteriosclerosis, chronic inflammatory processes, ischemia/reperfusion injury, and various neurodegenerative diseases [2]. The mitochondrial respiratory chain is the major source of intracellular ROS generation and, at the same time, an important target for the damaging effects of ROS (Fig. 1). Cellular metabolism depends on the continuous supply of ATP from the mitochondria, which in most tissues account for the bulk of ATP produced. Hence, any damage that impairs the function of the respiratory chain might also have an impact on cell viability. To protect cells from oxidative insult, mitochondria contain an elaborate defense system to detoxify ROS and repair ROS-induced damage.

Recent work on cell death mechanisms has placed mitochondria in the focus of apoptosis regulation. Generation of ROS, together with the release of pro-apoptotic proteins from the intermembrane space of mitochondria, trigger the activation of different modes of cell death. The effect of ROS 


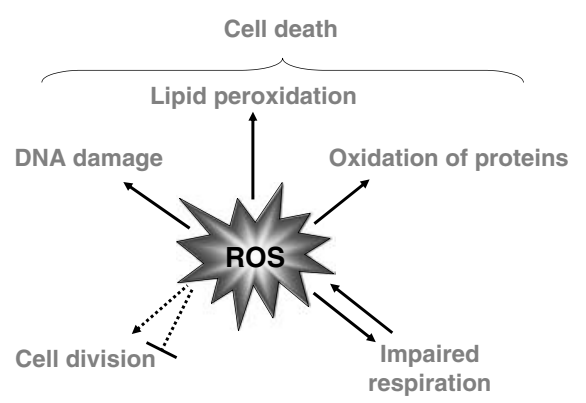

Fig. 1 Effects of reactive oxygen species on cellular functions and the induction of cell death. ROS induces DNA damage, oxidation of proteins, impairs mitochondrial respiration, and dose-dependently either stimulates or inhibits cellular proliferation

on cell viability and the induction of apoptosis is the topic of this review.

\section{Mitochondrial oxidative stress}

2.1 Mitochondria-The main source of ROS generation in aerobic cells

Oxidative stress is generally defined as an imbalance that favors the production of ROS over antioxidant defenses; however, the precise mechanisms by which ROS cause cellular injury remain elusive. As mentioned above, the majority of ROS are products of mitochondrial respiration. About 1-2\% of the molecular oxygen consumed during normal physiological respiration is converted into superoxide radicals. The one-electron reduction of molecular oxygen produces a relatively stable intermediate, the superoxide anion $\left(\mathrm{O}_{2}^{-}\right)$, which serves as the precursor of most ROS (Fig. 2). The dismutation of superoxide anions by superoxide dismutases results in $\mathrm{H}_{2} \mathrm{O}_{2}$ production. Subsequent interaction of $\mathrm{H}_{2} \mathrm{O}_{2}$ and $\mathrm{O}_{2}^{-}$in a Haber-Weiss reaction, or $\mathrm{Fe}^{2+}$ - $\left(\right.$ or $\left.\mathrm{Cu}^{2+}\right)$-driven cleavage of $\mathrm{H}_{2} \mathrm{O}_{2}$ in a Fenton reaction, can generate the highly reactive hydroxyl radical $(\mathrm{OH})$. The mitochondrial electron transport chain contains several redox centers that may leak electrons to molecular oxygen, serving as the primary source of superoxide production in most tissues. Superoxide-producing sites and enzymes were recently analyzed in detail in a comprehensive review [3].

There is growing evidence that most of the $\mathrm{O}_{2}^{--}$generated by intact mammalian mitochondria in vitro is produced by complex I. This $\mathrm{O}_{2}^{-}$production occurs primarily on the matrix side of the inner mitochondrial membrane (IMM) [4]. $\mathrm{O}_{2}^{-}$production by complex I was also found to be markedly stimulated in the presence of succinate, the substrate of Complex II [5]. In addition to Complex I, Complex III is regarded as an important site of $\mathrm{O}_{2}^{--}$production [6,7], especially when mitochondrial respiration is suppressed by antimycin, an inhibitor of complex III. $\mathrm{O}_{2}^{--}$produced at this site appears on both sides of the IMM [8]. Ubiquinone, a component of the mitochondrial respiratory chain, linking Complex I with III, and II with III, is regarded as a major player in the formation of $\mathrm{O}_{2}^{-}$by Complex III [9]. The oxidation of ubiquinone proceeds in a set of reactions known as the Q-cycle, and the unstable semiquinone is responsible for $\mathrm{O}_{2}^{-}$formation [10].

\subsection{Mitochondrial antioxidant defense systems}

Hence, the mitochondrial respiratory chain serves as a major source of ROS derived from the disproportionation of superoxide anions. Within the mitochondrial matrix, MnSOD converts superoxide to hydrogen peroxide, which can be further metabolized by glutathione peroxidase (Gpx1) and peroxiredoxin (PrxIII), or diffuse from the mitochondria into the cytosol (Fig. 2). Mitochondria contribute 20$30 \%$ of the cytosolic steady-state concentration of $\mathrm{H}_{2} \mathrm{O}_{2}$ [11]; $\mathrm{O}_{2}^{-}$can not cross biological membranes except in the protonated form, which constitutes only a very small fraction of the superoxide pool at physiological $\mathrm{pH}$ [12]. However, part of the $\mathrm{O}_{2}^{-}$generated during mitochondrial respiration can also be vectorially released into the intermembrane
Fig. 2 Formation, effects and inactivation of reactive oxygen species in mitochondria. GSH, reduced glutathione; GSSG, glutathione disulfide; Gpx, glutathione peroxidase; Grx, glutaredoxin; $\mathrm{IDH}_{\mathrm{m}}$, mitochondrial isocitrate dehydrogenase; NADP, nicotinamide adenine dinucleotide phosphate; Prx, peroxiredoxin; SOD, superoxide dismutase; $\mathrm{TH}$, transhydrogenase; Trx, thioredoxin, TrxR, thioredoxin reductase

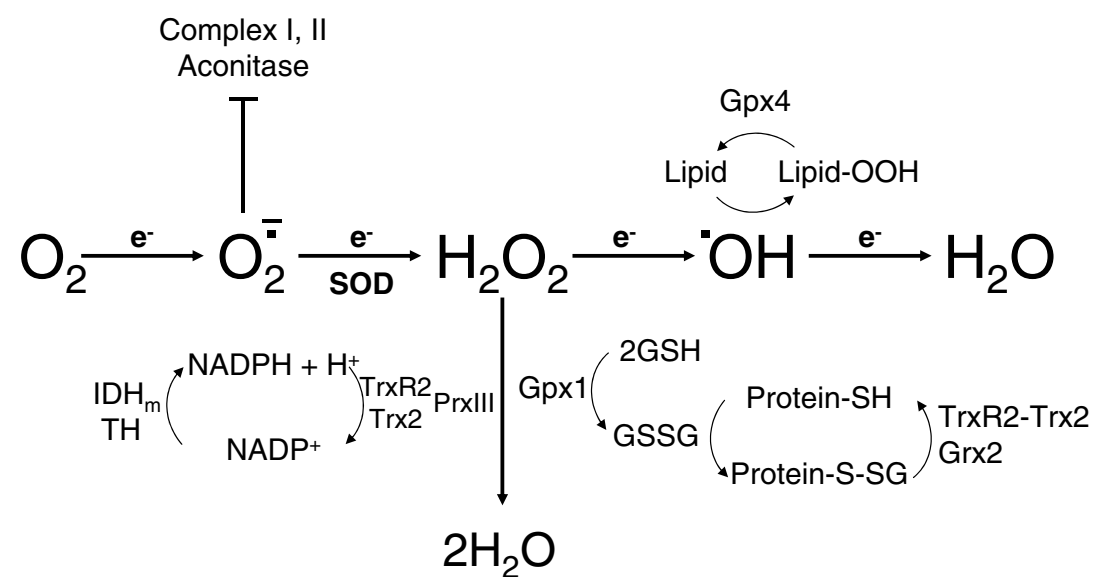


space [13]. At least in some cell types, CuZnSOD is present in the mitochondrial intermembrane space, where it can convert $\mathrm{O}_{2}^{-}$to $\mathrm{H}_{2} \mathrm{O}_{2}$ thus permitting further diffusion into the cytosol. Alternatively, when present in the intermembrane space, $\mathrm{O}_{2}^{-}$might be scavenged by cytochrome $c$ or diffuse into the cytosol through pores in the OMM, notably the voltage-dependent anion channel, VDAC [14]. Finally, under conditions of stimulated $\mathrm{NO}$ production $\mathrm{O}_{2}^{--}$might react with nitric oxide to form highly reactive peroxynitrite, $\mathrm{ONOO}^{-}$.

Most important for mitochondrial antioxidant protection is the tripeptide glutathione, GSH (L- $\gamma$-glutamyl-Lcysteinylglycine), and multiple GSH-linked antioxidant enzymes (Fig. 2). Although there is no evidence for GSH biosynthesis in mitochondria, these organelles have long been known to have their own GSH pool, which was early found to be critical for cell survival. Further support for the importance of this pool is provided by the finding that mitochondrial GSH was more resistant to depletion upon inhibition of GSH biosynthesis than the cytosolic GSH pool [15]. Among GSH-linked enzymes involved in mitochondrial antioxidant defense are Gpx1 and 4. These enzymes catalyze the reduction of $\mathrm{H}_{2} \mathrm{O}_{2}$, and of lipid hydroperoxides, with GSH as the electron donor. Gpx1 is the major isoform and is localized predominantly in the cytosol, but a small fraction is also present within the mitochondrial matrix. In contrast, Gpx4 (also known as phospholipid hydroperoxide glutathione peroxidase) is membrane-associated with a fraction localized to the mitochondria, possibly at the contact sites of the two membranes [16]. Gpx4 reduces hydroperoxide groups on phospholipids, lipoproteins and cholesteryl esters. Because of its small size and large hydrophobic surface it can interact with, and detoxify, membrane lipid hydroperoxides and does so much more efficiently than the alternative pathway, phospholipase $\mathrm{A}_{2}$ (PLA $)$-Gpx1 [17]. Therefore, Gpx4 is considered to be the primary enzymatic defense mechanism against oxidative damage to cellular membranes (Fig. 2). Finally, a newly discovered member of the family of GSH-linked mitochondrial redox enzymes is glutaredoxin 2 (Grx2), which was recently cloned and found to be present as both mitochondrial and nuclear isoforms [18]. Glutaredoxins catalyze glutathione-dependent dithiol reaction mechanisms, reducing protein disulfides, and monothiol reactions, reducing mixed disulfides between proteins and GSH (de/glutathionylation).

The mitochondrial thioredoxin system, which includes thioredoxin $2(\operatorname{Tr} x 2)$ and thioredoxin reductase $2(\operatorname{Tr} x R 2)$, is another potential source of disulfide reductase activity required for maintaining mitochondrial proteins in their reduced state (Fig. 2). Thioredoxins catalyze reduction of protein disulfides at much higher rates than Grx [19]. The thioredoxin system can also interact with the peroxiredoxins (Prx), which constitute a novel family of thiol-specific peroxidases that rely on Trx as the hydrogen donor for the reduction of hydrogen peroxide and lipid hydroperoxides [20]. One Prx isoform, Prx III, is exclusively detected in mitochondria [21].

\section{Consequences of oxidative stress}

Despite the presence of various antioxidants, e.g. ubiquinone and vitamin E, and antioxidant enzymes, the mitochondria appear to be the most powerful intracellular source of ROS; according to one estimate the steady state concentration of $\mathrm{O}_{2}^{-}$in the mitochondrial matrix is about 5- to 10-fold higher than that in the cytosol or nucleus [22]. Therefore, mitochondria might also be a primary target for the damaging effects of ROS. The interaction of diverse macromolecules with ROS may impair the function of these organelles and may directly influence cell viability and trigger cell death.

\subsection{Damage to DNA}

Oxidative damage to DNA causes modification of the purine and pyrimidine bases, the deoxyribose backbone, single and double strand breaks, as well as cross-links to other molecules (Fig. 1). DNA modifications are potentially mutagenic, contributing to cancer, premature ageing and neurodegenerative diseases [23].

One important target of ROS is the mitochondrial DNA (mtDNA), which encodes thirteen polypeptides, twenty-two transfer RNAs (tRNAs), and two ribosomal RNAs (rRNAs), all of which are essential for electron transport and ATP generation by oxidative phosphorylation [24]. This requires the assembly of the protein products of both the mitochondrial and nuclear genomes into functional respiratory complexes. mtDNA, therefore, represents a critical cellular target for oxidative damage that could lead to lethal cell injury through the disruption of electron transport, mitochondrial membrane potential, and ATP generation. mtDNA is especially susceptible to attack by ROS due to the close proximity to the electron transport chain, the major locus for free radical production, and the lack of protective histones. For example, mitochondrially-generated ROS can trigger the formation of 8-hydroxydeoxyguanosine, a lesion arising as a result of oxidative DNA damage; the level of oxidatively modified bases in mtDNA is 10 - to 20 -fold higher than that in nuclear DNA. Oxidative damage induced by ROS is probably a major source of mitochondrial genomic instability leading to respiratory dysfunction. This instability of mtDNA is thought to be one of the most important factors in ageing. 


\subsection{Damage to proteins}

An important mechanism of $\mathrm{O}_{2}^{-}$toxicity is the direct oxidation and inactivation of iron-sulfur $(\mathrm{Fe}-\mathrm{S})$ proteins, such as aconitases, and the associated release of iron [25] (Fig. 1). The inactivation of mitochondrial aconitase may have at least two major consequences. First, the formation of an inactive $[3 \mathrm{Fe}-4 \mathrm{~S}]^{+}$cluster results in the simultaneous release of $\mathrm{Fe}^{2+}$ and $\mathrm{H}_{2} \mathrm{O}_{2}$. In fact, $\mathrm{O}_{2}^{-}$-mediated inactivation of Fe-S-containing enzymes may pose a significant oxidative burden, because it provides equimolar amounts of $\mathrm{H}_{2} \mathrm{O}_{2}$ per mole of $\mathrm{O}_{2}^{-}$[26]. The release of $\mathrm{Fe}^{2+}$ and $\mathrm{H}_{2} \mathrm{O}_{2}$, ingredients of the Haber-Weiss and Fenton reactions, can also result in generation of the potent hydroxyl radical, which can oxidize mitochondrial proteins, DNA, and lipids, thereby amplifying $\mathrm{O}_{2}^{-}{ }^{-}$-initiated oxidative damage. Whether aconitase inactivation results in free radical formation and toxicity in vivo remains unknown.

Mitochondrial aconitase plays a key role in the Krebs cycle, catalyzing the conversion of citrate to isocitrate. Inhibition of mitochondrial aconitase, even partial, could result in Krebs cycle dysfunction and have an impact on energy production and cell viability. A recent study further documents that the mitochondrial aconitase is associated with protein-mtDNA complexes, called nucleoids. In this novel context, aconitase functions to stabilize mtDNA, perhaps by reversibly remodeling nucleoids to directly influence mitochondrial gene expression in response to changing cellular metabolism [27]. In addition, $\mathrm{O}_{2}^{--}$can inactivate several other iron-sulphur proteins, such as Complex I NADH dehydrogenase; $\mathrm{O}_{2}^{--}$revealed a mild efficiency towards succinate dehydrogenase $[22,28]$. Oxidized proteins are recognized by proteases and degraded; new replacement protein molecules have then to be synthesized de novo.

\subsection{Damage to lipids}

ROS formation and stimulation of lipid peroxidation in mitochondria can lead to suppression of mitochondrial metabolism (Fig. 1). Lipid peroxides affect vital mitochondrial functions, such as respiration and oxidative phosphorylation, inner membrane barrier properties, maintenance of mitochondrial membrane potential $(\Delta \psi)$, and mitochondrial $\mathrm{Ca}^{2+}$ buffering capacity [28-30]. Mitochondrial lipid peroxidation products can impair the barrier function of membranes by interacting either directly with the protein and/or indirectly with the lipid moieties of the membrane [31].

A potentially deleterious effect of ROS production in mitochondria is facilitation of $\mathrm{Ca}^{2+}$-dependent mitochondrial permeability transition (MPT), which plays a key role in certain modes of cell death (Fig. 3). In addition to ATP production in aerobic cells, mitochondria play a crucial role

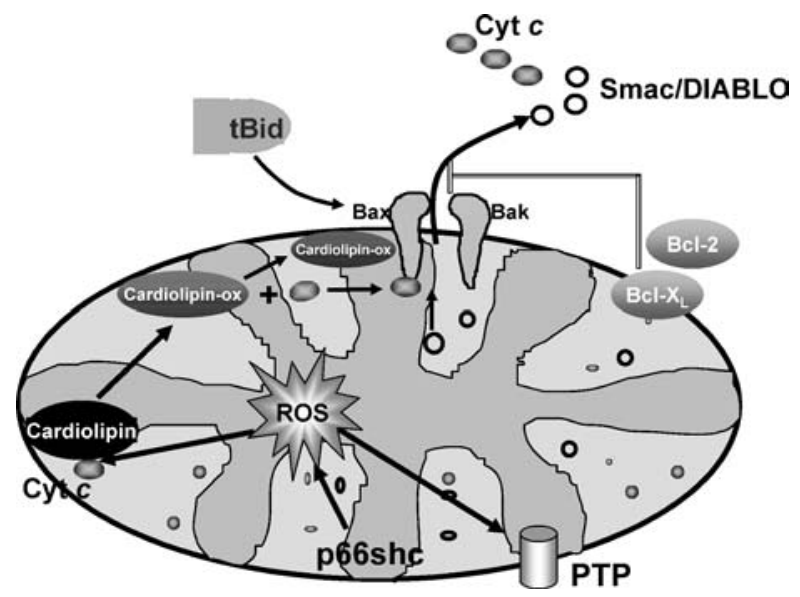

Fig. 3 Role of reactive oxygen species in mitochondrial control of cell death

in the regulation of intracellular $\mathrm{Ca}^{2+}$ homeostasis. Mitochondria can take up and retain $\mathrm{Ca}^{2+}$; however, the retention capacity is limited. If the accumulated $\mathrm{Ca}^{2+}$ exceeds a certain threshold-concentration, it is subsequently released from the mitochondria by the opening of a proteinaceous channel, commonly known as the permeability transition pore (PTP). The efflux of $\mathrm{Ca}^{2+}$ along with other matrix constituents disrupts the solute homeostasis, and causes drastic changes in mitochondrial ultrastructure and functional activity [32]. The $\mathrm{Ca}^{2+}$ threshold for MPT decreases when $\mathrm{Ca}^{2+}$ sequestration is accompanied by oxidative stress and depletion of adenine nucleotides. As oxidative stress markedly sensitizes mitochondria towards MPT induction, it was proposed that mitochondrially-generated ROS are directly involved in MPT induction [33]. Although not resolved in its detailed molecular composition, the prevailing hypothesis is that the MPT pore is a multimeric complex, composed of VDAC-ANT-CyP-D (voltage-dependent anion channeladenine nucleotide translocase-cyclophilin D). This complex is located at contact sites between the mitochondrial inner and outer membranes [34]. In addition, other proteins may bind to the pore complex, in particular kinases (e.g. hexokinase, creatine kinase). Recent studies identified the ANT as one important target for ROS induced by anticancer drugs, such as doxorubicin and arsenic trioxide. In particular, it was demonstrated that doxorubicin-induced cardiac toxicity correlates with oxidation of SH-groups in ANT and a decrease in ANT protein concentration, with inhibition of mitochondrial respiration and increased probability of MPT pore formation as consequences thereof [35] (Fig. 3). The role of VDAC as a target for ROS is less clear, although it has been demonstrated recently that $\mathrm{O}_{2}^{--}$modulates opening of VDAC reconstituted into liposomes, causing release of entrapped FITC-cytochrome $c$ [14].

Hence, oxidative stress and impaired $\mathrm{Ca}^{2+}$ homeostasis both contribute to mitochondrially-mediated cellular 
damage. MPT results in mitochondrial failure, which can lead to necrosis due to ATP depletion, or to caspase-mediated apoptosis if MPT induction occurs in a subpopulation of mitochondria and remaining organelles are still able to maintain their membrane potential and produce enough ATP to support the apoptotic process.

\subsection{Effect of cardiolipin peroxidation on cytochrome $c$ release}

Cytochrome $c$ is normally bound to the inner mitochondrial membrane by an association with the anionic phospholipid cardiolipin (Fig. 3). Cardiolipin is present only in mitochondria and is found primarily in the IMM. Because of its unique structure among phospholipids, cardiolipin confers fluidity and stability to the mitochondrial membrane. The molecular interaction between cardiolipin and cytochrome $c$ involves electrostatic interactions at the A-site of cytochrome $c$, whereas hydrophobic interactions and hydrogen bonding take place at its $\mathrm{C}$-site. To explain the latter binding mode, it was postulated that one of the acyl chains of cardiolipin may be inserted into a hydrophobic pore in cytochrome $c$, while the others extend into the phospholipid bilayer [36].

Since the bulk of cytochrome $c$ is bound to the IMM, it appears that the electrostatic and hydrophobic interactions between cardiolipin and cytochrome $c$ must be "breached" in order for cytochrome $c$ to leave the mitochondria. Apparently, simple permeabilization of the outer mitochondrial membrane (OMM) by oligomeric Bax in a low-ionic strength medium is insufficient for cytochrome $c$ release from mitochondria [37]. It was early found that cardiolipin oxidation decreases its binding affinity for cytochrome $c$ and, more recently, that oxidative modification of cardiolipin facilitates cytochrome $c$ mobilization from the IMM. Based on these results we hypothesized that cytochrome $c$ release during apoptosis occurs by a two-step process, involving first the detachment of the hemoprotein from the IMM, followed by permeabilization of the OMM and the release of cytochrome $c$ into the extramitochondrial milieu [37] (Fig. 3). These findings indicate that cardiolipin plays an important role not only in mitochondrial energy metabolism, but also in the retention of cytochrome $c$ within the intermembrane space.

Accordingly, accumulating data suggest that ROS facilitate the detachment of cytochrome $c$ from cardiolipin before its release into the soluble cytoplasm via pores in the OMM formed by pro-apoptotic proteins. Indeed, early studies utilizing bovine heart submitochondrial particles showed that mitochondrially-generated ROS decreased the content of cardiolipin in the membrane, and that this was concomitant with a decrease in the activity of cytochrome $c$ oxidase [38]. The cardiolipin content of the particles could be restored with exogenously added cardiolipin, but not with peroxidized cardiolipin. Studies of myocardial ischemia in the perfused rabbit heart have also revealed that increased ROS production leads to cardiolipin oxidation and depletion, combined with inhibition of Complex IV activity in subsarcolemmal mitochondria [39]. It is of interest to note that $\mathrm{PLA}_{2}$-mediated generation of lysocardiolipin was a late event, and that cardiolipin oxidation was primarily responsible for the depletion of cardiolipin seen in this model. In addition, Vogelstein's group reported that oxidative degradation of mitochondrial cardiolipin occurred during p53mediated apoptosis [40]. Furthermore, a model of glutamate toxicity in neurons demonstrated that cytochrome $c$ is released from mitochondria in a ROS-dependent fashion [41], and a burst of ROS in growth factor-deprived neurons was found to damage mitochondria by causing a profound loss of cardiolipin [42]. A host of more recent studies have also shown a correlation between preserved cardiolipin content and resistance to apoptosis upon manipulation of various mitochondrial antioxidant enzymes, such as PrxIII, Grx2 and Gpx4 (see discussion below).

The two-step concept of cytochrome $c$ release from mitochondria during apoptosis has been confirmed in several subsequent studies. For example, recent observations demonstrated that, in the absence of Complex I inhibitors, recombinant oligomeric Bax protein elicited only minimal cytochrome $c$ release $(\sim 18 \%)$ from brain mitochondria. However, when the mitochondria were incubated with both recombinant Bax and Complex I inhibitors, which were shown to stimulate ROS production and, hence, cardiolipin oxidation, up to $65 \%$ of the mitochondrial cytochrome $c$ was released. Thus, in accordance with the two-step concept, neither ROS production via Complex I inhibition, nor permeabilization of the OMM with Bax, alone, triggered overt release of cytochrome $c$, whereas their combination resulted in a marked release of the hemoprotein [43]. In another study, suppression of Complex I activity stimulated intramitochondrial oxidative stress, which, in turn, increased the releasable soluble pool of cytochrome $c$ within the mitochondrial intermembrane space [44]. Upon mitochondrial permeabilization by Bax, more cytochrome $c$ was released into the cytosol from brain mitochondria with impaired Complex I activity. Based on these results, the authors proposed a model in which defects in Complex I lower the threshold for activation of mitochondrially-mediated apoptosis by Bax, thereby rendering compromised neurons more prone to degenerate.

Selective peroxidation of cardiolipin was recently demonstrated by Kagan and colleagues to precede cytochrome $c$ release during apoptosis [45] (Fig. 3). Looking for the mechanism of cardiolipin oxidation, the authors found that cytochrome $c$, in complex with cardiolipin, catalyzes $\mathrm{H}_{2} \mathrm{O}_{2}$ dependent cardiolipin peroxidation which, in turn, facilitates the detachment of cytochrome $c$ from the outer surface 
of the IMM and its subsequent release into the cytosol through pores in the OMM. Quantitative characterization of the peroxidase activity of cytochrome $c$ revealed that at low ionic strength and high cardiolipin-cytochrome $c$ ratio, the peroxidase activity of the cardiolipin-cytochrome $c$ complex was increased more than 50-fold. This catalytic activity correlates with partial unfolding of cytochrome $c$, and an increase in the peroxidase activity preceded the loss of protein tertiary structure. It seems that electrostatic cardiolipincytochrome $c$ interactions are central to the initiation of the peroxidase activity, while hydrophobic interactions are involved when the tertiary structure of cytochrome $c$ is lost [46]. The finding that cytochrome $c$ might change its structure during apoptosis was unexpected, since several groups have shown that even small changes in the structure of cytochrome $c$ resulted in abrogation of its pro-apoptotic function [47]. When bound to cardiolipin-containing membranes, in vitro cytochrome $c$ peroxidase activity was stimulated at lower hydrogen peroxide concentrations compared to what was seen with cytochrome $c$ in the absence of phospholipid [46]. Altogether, this suggests that redistribution of cardiolipin within the mitochondrial membranes, combined with increased production of hydrogen peroxide, can switch on the peroxidase activity of cytochrome $c$ in mitochondria, and that cardiolipin oxidation might be a prerequisite step in the execution of apoptosis. The peroxidase function of the cardiolipin-cytochrome $c$ complex is compatible with the proposed two-step hypothesis of cytochrome $c$ release [37] and also provides an explanation for the anti-apoptotic effects reported for multiple mitochondrial antioxidant enzymes.

It is known that cells from Bax, Bak double-knockout mice are resistant to most inducers of apoptosis, and that the presence of these proteins is normally required for OMM permeabilization. However, their exact role in cardiolipin oxidation-mediated cytochrome $c$ release is still unclear. It was demonstrated, however, that mitochondrial production of ROS increased in the presence of Bax plus a BH3-domain peptide [48], apparently due to stimulated $\mathrm{O}_{2}^{--}$production by the respiratory chain following cytochrome $c$ release [49]. Increased ROS production may further contribute to cytochrome $c$ release by activating lipid peroxidation [50], thereby facilitating cytochrome $c$ dissociation from cardiolipin [51]. Clearly, the precise mechanism(s) of interaction of these proteins with oxidized cardiolipin remain to be investigated, as well as the nature of the promoting effect of cardiolipin hydroperoxides on OMM permeabilization. The interaction of $\mathrm{tBid}$ - the $\mathrm{C}$-terminal cleavage fragment of the pro-apoptotic Bcl-2 protein, Bid-with the cardiolipincytochrome $c$ complex also needs to be characterized (Fig. 3).

\section{Impact of oxidative stress on the regulation of apoptosis}

\subsection{Pro-oxidant molecular modulators of ROS}

One of the possible sources of ROS in mitochondria of apoptotic cells is the p66Shc protein, a redox enzyme that utilizes reducing equivalents derived from the mitochondrial electron transfer chain through the oxidation of cytochrome $c$ to produce $\mathrm{H}_{2} \mathrm{O}_{2}$ in the intermembrane space [52] (Fig. 3). Redoxdefective mutants of p66Shc are unable to induce mitochondrial ROS generation and swelling in vitro, or to mediate mitochondrial apoptosis in vivo. Interestingly, a fraction of this cytosolic enzyme localizes within mitochondria, where it forms a complex with mitochondrial Hsp70. Upon induction of apoptosis, dissociation of this complex is followed by the release of monomeric p66Shc and its interaction with cytochrome $c$ to generate hydrogen peroxide.

$\mathrm{p} 53$, a key player in the maintenance of genome integrity, is involved also in cellular redox regulation. Interaction of ROS with DNA can lead to DNA damage, which induces the stabilization of $\mathrm{p} 53$. The resulting increase in p53 activity triggers the expression of the Bcl-2 family proteins Bax and PUMA, which target the mitochondria to induce cytochrome $c$ release and subsequent activation of the caspase cascade. Mitochondria deficient in cytochrome $c$ may then generate ROS to amplify the initial insult. Taken together, p53 transactivation can lead to increased ROS production by engaging the mitochondrial branch of the apoptotic machinery. In addition, the activation of $\mathrm{p} 53$ and the type of cell fate initiated was shown to be influenced by the cellular redox state and by mitochondrial activity [53]. Depending on the protein level of p53, and on the level of PUMA and Bax, ROS accumulation may lead to the induction of either apoptosis or senescence.

Recently, two other p53-regulated genes were discovered and found to have an impact on cellular ROS homeostasis and mitochondrial bioenergetics. TIGAR (Tp53-induced glycolysis and apoptosis regulator), a homologue of fructose-2,6biphosphatase, was shown to be upregulated by p53 [54]. This, in turn, leads to inhibition of glycolysis, allowing a higher flux of glucose into the pentose phosphate branch with increases in intracellular NADPH and GSH levels as a result. This sequence of events might serve as a mechanism of protection against ROS and apoptosis. Accordingly, downregulation of TIGAR expression by RNAi led to accelerated cell death induced by ROS. These new findings further contribute to our understanding of how redox modulation influences cellular metabolism and apoptosis.

The other p53-regulated protein recently identified is $\mathrm{SCO} 2$, an assembly factor for cytochrome oxidase that helps 
the mitochondrially-encoded subunit Cox2 to be incorporated into the COX complex [55]. Hence, p53 mutations can lead to the suppression of mitochondrial respiration by direct effects on the biogenesis of mitochondrial respiratory chain components. This finding could also help explain how respiration is lowered in favor of glycolytic ATP generation in cancer cells where p53 is often mutated. This phenomenon is known since more than 80 years as the Warburg effect [56].

\subsection{Antioxidant enzymes as anti-apoptotic devices}

As discussed above, mitochondrial ROS production seems to be an integrated part of both apoptotic and necrotic cell death. Accordingly, protection from oxidative stress by administration of antioxidants, such as vitamin $\mathrm{E}$ and ubiquinone, has been found to increase cell viability in multiple experimental model systems [13]. Also under normal physiological conditions cell viability and function are critically dependent on the maintenance of a proper balance between mitochondrial ROS generation and inactivation.

The importance of a tight regulation of the mitochondrial redox balance is further emphasized by the broad spectrum of antioxidant enzymes present in the mitochondria, and the fact that deletion of several of these enzymes is incompatible with cell viability and also causes embryonic lethality. For example, disruption of the $M n S O D$ gene results in early postnatal lethality, while $\mathrm{CuZnSOD}{ }^{-/-}$mice show no apparent phenotypic alterations [57]. This difference seems to be directly related to the different subcellular localization of the SODs, since MnSOD deletion can be compensated for by the targeting of CuZnSOD into the mitochondrial matrix. Further, disruption of the mitochondrial thioredoxin system $\left(\operatorname{Tr} \times 2^{-/-}\right)$also confers embryonic lethality [58], whereas $G p x I^{-/-}$mice are healthy, except under conditions of extreme oxidative stress. The latter finding may be due to the fact that PrxIII is more important for mitochondrial $\mathrm{H}_{2} \mathrm{O}_{2}$ catabolism than Gpx1. In contrast, disruption of the Gpx4 gene causes embryonic lethality, and cell lines from $\mathrm{Gpx}^{+/-}$ mice are hypersensitive to $\mathrm{H}_{2} \mathrm{O}_{2}$ and other agents that cause oxidative stress [59].

A large number of studies have been devoted to effects of modulation of mitochondrial antioxidant enzymes on cell viability and susceptibility to apoptotic cell death. Heterozygous $M n S O D$ knockout mice exhibit numerous alterations in mitochondrial function, including inhibition of aconitase and Complex I and II of the respiratory chain, enhanced susceptibility to induction of MPT and increased lipid peroxidation [60]. In contrast, overexpression of MnSOD attenuates mitochondrial ROS generation, protects respiratory function and blocks apoptosis in some experimental models. Although $\mathrm{O}_{2}^{--}$in the mitochondrial matrix has been proposed to activate uncoupling proteins (UCP), thereby providing a feedback mechanism that would limit further $\mathrm{O}_{2}^{--}$production by the respiratory chain, studies of $M n S O D$ transgenic mice have failed to provide evidence for altered UCP activities [61].

As mentioned above, disruption of the $\operatorname{Tr} x 2$ gene results in a lethal embryonic phenotype that is associated with massive apoptosis during early embryogenesis [58]. Trx2-deficient chicken B-cells were also reported to undergo apoptosis in the absence of exogenous triggers and to be hypersensitive to treatment with agents that induce oxidative stress [62]. Similar results have been obtained in experiments with Grx2, in which Grxz overexpressing HeLa cells were found to be less susceptible to mitochondrially-mediated apoptosis [63], while glutaredoxin 2 deficient cells were hypersensitive to triggers of oxidative cell damage [64]. The Grx system has also recently been suggested to be involved in the antiapoptotic effect of $17-\beta$-estradiol in $\mathrm{H}_{2} \mathrm{O}_{2}$-treated cardiac H9c2 cells [65].

Mitochondrial PrxIII is also dependent on the mitochondrial thioredoxin system for activity and is probably the most important $\mathrm{H}_{2} \mathrm{O}_{2}$ metabolizing system in these organelles. A recent study in HeLa cells has shown that knockdown of PrxIII using RNA interference technology resulted in increased intracellular levels of $\mathrm{H}_{2} \mathrm{O}_{2}$ and sensitized the cells to induction of mitochondrially-mediated apoptosis by staurosporine or TNF [66]. These effects were reversed by ectopic expression of PrxIII or mitochondrially-targeted catalase. PrxIII was found to be more abundant than Gpx1, and the study supports the hypothesis that PrxIII is the most important $\mathrm{H}_{2} \mathrm{O}_{2}$ scavenging enzyme in the mitochondria.

Gpx4, also known as phospholipid hydroperoxide glutathione peroxidase, represents the most extensively studied enzyme involved in mitochondrial antioxidant defense and protection from apoptosis. Gpx4 null mice die in utero by mid-gestation, and $G p x 4^{+/-}$mice show reduced survival upon $\gamma$-irradiation [59]. Cell lines from these animals are extremely vulnerable to oxidative injury, whereas transgenic mice overexpressing Gpx4 demonstrated increased resistance to diquat-induced liver injury and to apoptosis induced by oxidative stress [67]. Protection from apoptosis was early reported to be specific for overexpression of the mitochondrial form of Gpx4 and to be related to suppression of the mitochondrial pathway of apoptosis signaling [68]. More recent findings have linked the inhibitory effect of Gpx4 on apoptosis to a protection of the adenine nucleotide translocase from the loss of activity that is otherwise associated with apoptosis, possibly by inhibition of cardiolipin oxidation [69]. It is of interest to note that the anti-apoptotic effects of PrxIII, Grx2, and Gpx4 have all been related to a possible protection of cardiolipin from peroxidation during the early stage of apoptosis [63, 66, 69].

Thus, it appears that the mitochondrial antioxidant defense systems are of critical importance for the regulation of apoptosis. Although further work is certainly required to characterize the mechanism(s) of protection, inhibition of 
cardiolipin oxidation during the early apoptotic phase might contribute to protection. It is also remarkable that there seems to be so little functional overlap between the various systems, and that modulation of the activity of one particular defense system is directly reflected on cellular susceptibility to undergo apoptosis.

\section{Summary}

Research during the last decades has revealed multiple defense systems that allow the eukaryotic cell to deal with the highly toxic oxygen radicals. Not surprisingly, oxidative stress is a conserved signal for cell death and is involved in a variety of cell death paradigms. Hence, small molecules like ROS can impact on the complex networks of proteins mediating the induction and execution of cell death (Fig. 1). Molecular damage elicited by ROS challenges the cells to either repair the damage or, if this is not possible, to activate the cell death programme. Mitochondria, which are the switchboard of the apoptosis machinery, serve as the prime source of ROS as well as a target for their damaging effects. In fact, the respiratory chain generates the majority of cellular ROS, which can then interact directly with mitochondrial proteins, lipids and DNA, obscuring their functions and the operation of the organelles. Lipid peroxidation, notably the peroxidation of cardiolipin, appears to be a critical early event in apoptosis. Oxidatively modified cardiolipin looses its affinity for cytochrome $c$ binding, leading to the accumulation of "free" cytochrome $\mathrm{c}$ in the intermembrane space, which is subsequently released into the cytosol upon permeabilization of the OMM. To protect from oxidative damage, mitochondria possess multiple antioxidant defense systems. It has now been convincingly shown that modulation of these systems directly affects the susceptibility of cells to undergo apoptosis. The study of how ROS influence the activation of the cell death programme may therefore continue to reveal mechanisms, which might be used for therapeutic intervention in major human diseases.

Acknowledgment Work in the authors' laboratory was supported by grants from the Swedish (3829-B05-10XBC) and Stockholm (061491) Cancer Societies, The Swedish Research Council (K2006-31X-0247139-3 and 2006-3970), the European Commission (ONCODEATH037278), "Gosta Fraenckel's Foundation for Medical research". MO is a recipient of the Wenner-Gren Foundation Fellowship. We apologize to authors whose primary references could not be cited due to space limitations.

\section{References}

1. Madeo F, Frohlich E, Ligr M et al (1999) Oxygen stress: a regulator of apoptosis in yeast. J Cell Biol 145:757-767
2. Droge W (2002) Free radicals in the physiological control of cell function. Physiol Rev 82:47-95

3. Andreyev AY, Kushnareva YE, Starkov AA (2005) Mitochondrial metabolism of reactive oxygen species. Biochemistry (Mosc) 70:200-214

4. de Vries S (1986) The pathway of electron transfer in the dimeric QH2: cytochrome c oxidoreductase. J Bioenerg Biomembr 18:195-224

5. Liu Y, Fiskum G, Schubert D (2002) Generation of reactive oxygen species by the mitochondrial electron transport chain. J Neurochem 80:780-787

6. Grigolava IV, Ksenzenko M, Konstantinob AA, Tikhonov AN, Kerimov TM (1980) Tiron as a spin-trap for superoxide radicals produced by the respiratory chain of submitochondrial particles. Biokhimiia 45:75-82

7. Turrens JF, Boveris A (1980) Generation of superoxide anion by the NADH dehydrogenase of bovine heart mitochondria. Biochem J 191:421-427

8. Muller FL, Liu Y, Van Remmen H (2004) Complex III releases superoxide to both sides of the inner mitochondrial membrane. J Biol Chem 279:49064-49073

9. Rich PR, Bonner WD (1978) The sites of superoxide anion generation in higher plant mitochondria. Arch Biochem Biophys 188:206-213

10. Turrens JF, Alexandre A, Lehninger AL (1985) Ubisemiquinone is the electron donor for superoxide formation by complex III of heart mitochondria. Arch Biochem Biophys 237:408-414

11. Han D, Antunes F, Canali R, Rettori D, Cadenas E (2003) Voltage-dependent anion channels control the release of the superoxide anion from mitochondria to cytosol. J Biol Chem 278: 5557-5563

12. Gus'kova RA, Ivanov, II, Kol'tover VK, Akhobadze VV, Rubin AB (1984) Permeability of bilayer lipid membranes for superoxide $\left(\mathrm{O}_{2}{ }^{-}\right)$radicals. Biochim Biophys Acta 778:579-585

13. Fariss MW, Chan CB, Patel M, van Houten B, Orrenius S (2005) Role of mitochondria in toxic oxidative stress. Mol Interven 5:98-114

14. Madesh M, Hajnoczky G (2001) VDAC-dependent permeabilization of the outer mitochondrial membrane by superoxide induces rapid and massive cytochrome $\mathrm{c}$ release. J Cell Biol 155:1003-1015

15. Soderdahl T, Enoksson M, Lundberg M et al (2003) Visualization of the compartmentalization of glutathione and protein-glutathione mixed disulfides in cultured cells. Faseb J 17:124-126

16. Pushpa-Rekha TR, Burdsall AL, Oleksa LM, Chisolm GM, Driscoll DM (1995) Rat phospholipid-hydroperoxide glutathione peroxidase. cDNA cloning and identification of multiple transcription and translation start sites. J Biol Chem 270: 26993-26999

17. Antunes F, Salvador A, Pinto RE (1995) PHGPx and phospholipase A2/GPx: comparative importance on the reduction of hydroperoxides in rat liver mitochondria. Free Radic Biol Med 19:669-677

18. Lundberg M, Johansson C, Chandra J et al (2001) Cloning and expression of a novel human glutaredoxin (Grx2) with mitochondrial and nuclear isoforms. J Biol Chem 276:26269-26275

19. Arner ES, Holmgren A (2000) Physiological functions of thioredoxin and thioredoxin reductase. Eur J Biochem 267:6102-6109

20. Chae HZ, Kang SW, Rhee SG (1999) Isoforms of mammalian peroxiredoxin that reduce peroxides in presence of thioredoxin. Methods Enzymol 300:219-226

21. Watabe S, Hiroi T, Yamamoto Y, et al (1997) SP-22 is a thioredoxin-dependent peroxide reductase in mitochondria. Eur $\mathrm{J}$ Biochem 249:52-60

22. Cadenas E, Davies KJ (2000) Mitochondrial free radical generation, oxidative stress, and aging. Free Radic Biol Med 29:222-230 
23. Bohr VA (2002) Repair of oxidative DNA damage in nuclear and mitochondrial DNA, and some changes with aging in mammalian cells. Free Radic Biol Med 32:804-812

24. Anderson S, Bankier AT, Barrell BG et al (1981) Sequence and organization of the human mitochondrial genome. Nature 290:457-465

25. Fridovich I (1997) Superoxide anion radical $\left(\mathrm{O}_{2}{ }^{-}\right)$, superoxide dismutases, and related matters. J Biol Chem 272:18515-18517

26. Liochev SI, Fridovich I (1999) The relative importance of HO* and ONOO- in mediating the toxicity of $\mathrm{O}^{*}$. Free Radic Biol Med 26:777-778

27. Chen XJ, Wang X, Kaufman BA, Butow RA (2005) Aconitase couples metabolic regulation to mitochondrial DNA maintenance. Science 307:714-717

28. Zhang Y, Marcillat O, Giulivi C, Ernster L, Davies KJ (1990) The oxidative inactivation of mitochondrial electron transport chain components and ATPase. J Biol Chem 265:16330-16336

29. Albano E, Bellomo G, Parola M, Carini R, Dianzani MU (1991) Stimulation of lipid peroxidation increases the intracellular calcium content of isolated hepatocytes. Biochim Biophys Acta 1091:310-316

30. Bacon BR, O'Neill R, Britton RS (1993) Hepatic mitochondrial energy production in rats with chronic iron overload. Gastroenterology 105:1134-1140

31. Chen JJ, Bertrand H, Yu BP (1995) Inhibition of adenine nucleotide translocator by lipid peroxidation products. Free Radic Biol Med 19:583-590

32. Orrenius S, Zhivotovsky B, Nicotera P (2003) Regulation of cell death: the calcium-apoptosis link. Nat Rev Mol Cell Biol 4:552-565

33. Kowaltowski AJ, Castilho RF, Vercesi AE (1996) Opening of the mitochondrial permeability transition pore by uncoupling or inorganic phosphate in the presence of $\mathrm{Ca}^{2+}$ is dependent on mitochondrial-generated reactive oxygen species. FEBS Lett 378:150-152

34. Beutner G, Ruck A, Riede B, Welte W, Brdiczka D (1996) Complexes between kinases, mitochondrial porin and adenylate translocator in rat brain resemble the permeability transition pore. FEBS Lett 396:189-195

35. Oliveira PJ, Wallace KB (2006) Depletion of adenine nucleotide translocator protein in heart mitochondria from doxorubicintreated rats-relevance for mitochondrial dysfunction. Toxicology 220:160-168

36. Tuominen EK, Wallace CJ, Kinnunen PK (2002) Phospholipidcytochrome $c$ interaction: evidence for the extended lipid anchorage. J Biol Chem 277:8822-8826

37. Ott M, Robertson JD, Gogvadze V, Zhivotovsky B, Orrenius S (2002) Cytochrome $c$ release from mitochondria proceeds by a two-step process. Proc Natl Acad Sci USA 99:1259-1263

38. Paradies G, Petrosillo G, Pistolese M, Ruggiero FM (2000) The effect of reactive oxygen species generated from the mitochondrial electron transport chain on the cytochrome $c$ oxidase activity and on the cardiolipin content in bovine heart submitochondrial particles. FEBS Lett 466:323-326

39. Petrosillo G, Di Venosa N, Ruggiero FM et al (2005) Mitochondrial dysfunction associated with cardiac ischemia/reperfusion can be attenuated by oxygen tension control. Role of oxygen-free radicals and cardiolipin. Biochim Biophys Acta 1710:78-86

40. Polyak K, Xia Y, Zweier JL, Kinzler KW, Vogelstein B (1997) A model for p53-induced apoptosis. Nature 389:300-305

41. Manna SK, Zhang HJ, Yan T, Oberley LW, Aggarwal BB (1998) Overexpression of manganese superoxide dismutase suppresses tumor necrosis factor-induced apoptosis and activation of nuclear transcription factor-kappaB and activated protein-1. J Biol Chem 273:13245-13254

42. Atlante A, Calissano P, Bobba A, Azzariti A, Marra E, Passarella
$\mathrm{S}$ (2000) Cytochrome $c$ is released from mitochondria in a reactive oxygen species (ROS)-dependent fashion and can operate as a ROS scavenger and as a respiratory substrate in cerebellar neurons undergoing excitotoxic death. J Biol Chem 275:37159-37166

43. Piccotti L, Buratta M, Giannini S, Gresele P, Roberti R, Corazzi L (2004) Binding and release of cytochrome $c$ in brain mitochondria is influenced by membrane potential and hydrophobic interactions with cardiolipin. J Membr Biol 198:43-53

44. Perier C, Tieu K, Guegan C et al (2005) Complex I deficiency primes Bax-dependent neuronal apoptosis through mitochondrial oxidative damage. Proc Natl Acad Sci USA 102:19126-19131

45. Kagan VE, Tyurin VA, Jiang J et al (2005) Cytochrome $c$ acts as a cardiolipin oxygenase required for release of proapoptotic factors. Nat Chem Biol 1:223-232

46. Belikova NA, Vladimirov YA, Osipov AN et al (2006) Peroxidase activity and structural transitions of cytochrome $c$ bound to cardiolipin-containing membranes. Biochemistry 45:4998-5009

47. Hampton MB, Zhivotovsky B, Slater AF, Burgess DH, Orrenius S (1998) Importance of the redox state of cytochrome c during caspase activation in cytosolic extracts. Biochem J 329(Pt 1):95-99

48. Starkov AA, Polster BM, Fiskum G (2002) Regulation of hydrogen peroxide production by brain mitochondria by calcium and Bax. J Neurochem 83:220-228

49. Cai J, Jones DP (1998) Superoxide in apoptosis. Mitochondrial generation triggered by cytochrome $c$ loss. J Biol Chem 273:11401-11404

50. Kirkland RA, Adibhatla RM, Hatcher JF, Franklin JL (2002) Loss of cardiolipin and mitochondria during programmed neuronal death: evidence of a role for lipid peroxidation and autophagy. Neuroscience 115:587-602

51. Shidoji Y, Hayashi K, Komura S, Ohishi N, Yagi K (1999) Loss of molecular interaction between cytochrome $\mathrm{c}$ and cardiolipin due to lipid peroxidation. Biochem Biophys Res Commun 264:343-347

52. Giorgio M, Migliaccio E, Orsini F et al (2005) Electron transfer between cytochrome $c$ and p66Shc generates reactive oxygen species that trigger mitochondrial apoptosis. Cell 122:221-233

53. Macip S, Igarashi M, Berggren P, Yu J, Lee SW, Aaronson SA (2003) Influence of induced reactive oxygen species in p53-mediated cell fate decisions. Mol Cell Biol 23:8576-8585

54. Bensaad K, Tsuruta A, Selak MA et al (2006) TIGAR, a p53inducible regulator of glycolysis and apoptosis. Cell 126:107-120

55. Matoba S, Kang JG, Patino WD et al (2006) p53 regulates mitochondrial respiration. Science 312:1650-1653

56. Warburg O (1956) On respiratory impairment in cancer cells. Science 124:269-270

57. Li Y, Huang TT, Carlson EJ et al (1995) Dilated cardiomyopathy and neonatal lethality in mutant mice lacking manganese superoxide dismutase. Nat Genet 11:376-381

58. Nonn L, Williams RR, Erickson RP, Powis G (2003) The absence of mitochondrial thioredoxin 2 causes massive apoptosis, exencephaly, and early embryonic lethality in homozygous mice. Mol Cell Biol 23:916-922

59. Yant LJ, Ran Q, Rao L et al (2003) The selenoprotein GPX4 is essential for mouse development and protects from radiation and oxidative damage insults. Free Radic Biol Med 34:496-502

60. Melov S, Coskun P, Patel M et al (1999) Mitochondrial disease in superoxide dismutase 2 mutant mice. Proc Natl Acad Sci USA 96:846-851

61. Mattiasson G, Shamloo M, Gido G et al (2003) Uncoupling protein-2 prevents neuronal death and diminishes brain dysfunction after stroke and brain trauma. Nat Med 9:1062-1068

62. Tanaka T, Hosoi F, Yamaguchi-Iwai Y et al (2002) Thioredoxin-2 (TRX-2) is an essential gene regulating mitochondria-dependent apoptosis. Embo J 21:1695-1703

63. Enoksson M, Fernandes AP, Prast S, Lillig CH, Holmgren A, Orrenius S (2005) Overexpression of glutaredoxin 2 attenuates 
apoptosis by preventing cytochrome $c$ release. Biochem Biophys Res Commun 327:774-779

64. Lillig CH, Lonn ME, Enoksson M, Fernandes AP, Holmgren A (2004) Short interfering RNA-mediated silencing of glutaredoxin 2 increases the sensitivity of HeLa cells toward doxorubicin and phenylarsine oxide. Proc Natl Acad Sci USA 101:13227-13232

65. Urata Y, Ihara Y, Murata H et al (2006) 17beta-estradiol protects against oxidative stress-induced cell death through the glutathione/glutaredoxin-dependent redox regulation of Akt in myocardiac H9c2 cells. J Biol Chem 281:13092-13102

66. Chang TS, Cho CS, Park S, Yu S, Kang SW, Rhee SG (2004) Peroxiredoxin III, a mitochondrion-specific peroxidase, regulates apoptotic signaling by mitochondria. J Biol Chem 279:41975-41984
67. Ran Q, Liang H, Gu M et al (2004) Transgenic mice overexpressing glutathione peroxidase 4 are protected against oxidative stress-induced apoptosis. J Biol Chem 279: 55137-55146

68. Nomura K, Imai H, Koumura T, Arai M, Nakagawa Y (1999) Mitochondrial phospholipid hydroperoxide glutathione peroxidase suppresses apoptosis mediated by a mitochondrial death pathway. J Biol Chem 274:29294-29302

69. Nomura K, Imai H, Koumura T, Kobayashi T, Nakagawa Y (2000) Mitochondrial phospholipid hydroperoxide glutathione peroxidase inhibits the release of cytochrome $c$ from mitochondria by suppressing the peroxidation of cardiolipin in hypoglycaemia-induced apoptosis. Biochem J 351:183-193 